\title{
Correction to: Is there a relation between sudden sensorineural hearing loss and white matter lesions?
}

\author{
Massimo Fusconi ${ }^{1}$. Giuseppe Attanasio ${ }^{1}$ Flavia Capitani ${ }^{2}$ (D) Edoardo Di Porto ${ }^{3}$. Davide Diacinti ${ }^{4,5}$. Isotta Musy ${ }^{1}$. \\ Massimo Ralli ${ }^{1}$. Giovanni Ralli ${ }^{1}$. Antonio Greco ${ }^{1} \cdot$ Marco de Vincentiis $^{6} \cdot$ Claudio Colonnese $^{7,8}$
}

Published online: 10 September 2019

๑) Springer-Verlag GmbH Germany, part of Springer Nature 2019

\section{Correction to: European Archives of Oto-Rhino-Laryngology https://doi.org/10.1007/s00405-019-05593-4}

In the original publication, fifth author's surname was incorrectly published as "Diacinto". The correct surname should read as "Diacinti".

The original article has been corrected.

Publisher's Note Springer Nature remains neutral with regard to jurisdictional claims in published maps and institutional affiliations.

The original article can be found online at https://doi.org/10.1007/ s00405-019-05593-4.

Flavia Capitani

fla.capitani@yahoo.it

1 Department of Sense Organs, Sapienza University of Rome, Rome, Italy

2 Clinic for Ear, Nose and Throat Medicine, Uniklinik of Tuebingen, Albstrasse 93, 70597 Stuttgart, Germany

3 Department of Economics and Statistics, University of Naples Federico II, Complesso Universitario di Monte Sant'Angelo, Naples, Italy

4 Department of Radiological Sciences, Oncology and Anatomo-Pathology, University Sapienza, Rome, Italy

5 Department of Odontostomatological and Maxillo-Facial Sciences, Umberto I Hospital, University Sapienza, Rome, Italy

6 Department of Oral and Maxillofacial Sciences, Sapienza University of Rome, Rome, Italy

7 Department of Neurology and Psichiatry, Neuroradiology Section, University Sapienza, Rome, Italy

8 IRCCS Neuromed, Pozzilli, IS, Italy 